\title{
Evaluation of the adherence to subcutaneous immunotherapy in patients with pollen allergy in usual clinical practice: The CITY study
}

Francisco Javier Solá Martínez ${ }^{1}$, María Luisa González Gutiérrez ${ }^{2}$, María Teresa Palomeque Rodríguez ${ }^{3}$, Emilio Funes Vera ${ }^{4}$, Ana Montoro De Francisco ${ }^{5}$, Elena Laffond Yges ${ }^{6}$, Dolores Hernández Fernández de Rojass, Matilde Rodríguez Mosquera ${ }^{7}$, Lourdes Pérez González ${ }^{8}$, Stephan Cimbollek ${ }^{9}$, Ana María Navarro Pulido ${ }^{10}$, Nataly Cancelliere ${ }^{11}$, Begoña Soler López ${ }^{12 *}$ and Javier Cuesta Herranz ${ }^{13}$

${ }^{1}$ Hospital Universitario Ramón y Cajal, Madrid, Spain

${ }^{2}$ Hospital Clínico San Carlos, Madrid, Spain

${ }^{3}$ Hospital Universitario Nuestra Señora del Perpetuo Socorro, Albacete, Spain

${ }^{4}$ Hospital Rafael Méndez, Lorca (Murcia), Spain

${ }^{5}$ Hospital Central de la Defensa Gómez Ulla, Madrid, Spain

${ }^{6}$ Hospital Virgen de la Vega, Salamanca, Spain

${ }^{7}$ Hospital Universitario La Fe, Valencia, Spain

${ }^{8}$ Hospital Universitario Puerta de Hierro, Majadahonda (Madrid), Spain

${ }^{9}$ Hospital Virgen del Rocío, Sevilla, Spain

${ }^{10}$ Hospital El Tomillar, Sevilla, Spain

${ }^{11}$ Merck, S.L.U., Madrid, Spain

${ }^{12}$ E-C-BIO, S.L., Madrid, Spain

${ }^{13}$ Fundación Jiménez Díaz, Madrid, Spain

\begin{abstract}
Background: The objective of the study was to determine patient compliance and adherence to treatment with Allergen Immunotherapy (AIT) with Allergovit ${ }^{\circ}$ for pollen allergic rhinitis and/or bronchial asthma.

Methods: A retrospective observational study was designed, included pollen allergic patients with rhinoconjunctivitis and/or bronchial asthma treated with subcutaneous immunotherapy with Allergovit ${ }^{\circ}$. Compliance with treatment was defined as the ratio between administered and prescribed doses. Compliance was considered adequate when higher than $80 \%$. Adherence was evaluated from the number of patients withdrawing from the treatment during 3-year follow-up. Causes for non-adherence, symptoms evolution and the physician Clinical Global Impression score were also documented.

Results: A total of 176 patients from 9 Allergy Units were included, 44.4\% ( $\mathrm{n}=79)$ males; mean age 33.8 years (95\%CI 31.9-35.7). AIT administration was preseasonal in 19.9\% $(\mathrm{n}=32)$, pre-co-seasonal in $2.5 \%(\mathrm{n}=4)$ and perennial in $77.6 \%(\mathrm{n}=125)$. The presence and frequency of ocular, nasal and bronchial symptoms decreased significantly during follow-up ( $\mathrm{p}<0.0001$ ). Mean compliance with treatment in years 1,2 and 3 was: $98.6 \%, 98.7 \%$ and $96.5 \%$. Compliance was adequate in years 1,2 and 3 in $95.9 \%, 95 \%$ and $96.2 \%$ of the cases. The three years of immunotherapy were completed by 158 patients (89.8\%). Reasons for non-adherence were mainly symptoms improvement and the appearance of adverse reactions. The latter were observed in 34 patients (19.3\%), with 40 adverse reactions during three years of follow-up: Thirty-three reactions were local and seven were systemic.
\end{abstract}

Conclusions: Observed compliance and adherence to AIT immunotherapy was very high, throughout the three-year follow-up period, and the treatment showed an adequate safety profile.

Abbreviations: AIT: Allergen immunotherapy; ANOVA: Analysis of variance; CI: Confidence interval; SEAIC: Sociedad española de alergología e inmunología clínica; VAS: Visual analog scale; WHO: World health organization.
${ }^{*}$ Correspondence to: Begoña Soler López, Medical Director, E-C-BIO, S.L., c/ Rosa de Lima, 1, Edificio ALBA, Office 016, 28230 - Las Rozas (Madrid), Spain, Tel: +34 9163004 80; Fax: +34 9185829 00; E-mail: bsoler@ecbio.net

Key words: immunotherapy, adherence, compliance, subcutaneous, allergoid, pollen

Received: May 07, 2019; Accepted: June 14, 2019; Published: June 17, 2019 


\section{Introduction}

Allergic diseases constitute an increasingly important health problem of pandemic proportions in Europe. It is estimated that over 150 million people are currently affected [1]. Thirty percent of the European population suffers from rhinitis and/or allergic conjunctivitis, and $20 \%$ presents from bronchial asthma.

According to the therapeutic guidelines [2-5], the management of these allergic diseases is based on three fundamental principles: avoidance of the causal allergen; treatment of the symptoms (drug therapy); and specific etiological treatment with specific Allergen Immunotherapy (AIT) with allergens.

Four meta-analyses have documented subcutaneous AIT efficacy in the management of bronchial asthma [6-9]. The meta-analysis carried out by Calderón et al. documented its efficacy for the treatment of allergic rhinitis [10]. Among the studies that evidence the safety of AIT in our setting, special mention must be made of the trial carried out by the Immunotherapy Committee of the Spanish society of Allergology and Clinical Immunology (Sociedad Española de Alergología e Inmunología Clínica, SEAIC), under conditions of usual clinical practice [11].

When compared with pharmacological treatment, the main advantages of AIT are that it is specific and etiological, offers longterm efficacy after completion of the treatment cycle, and is capable of preventing new sensitizations to other allergens in the treated patients [12].

Allergovit is a hypoallergenic depot formulation indicated for the treatment of IgE-mediated allergic rhinitis and/or bronchial asthma secondary to pollen hypersensitivity. The hypoallergenicity of the formulation makes it possible to administer higher allergen maintenance doses within the upper range of the interval recommended by the World Health Organization (WHO) [5], in order to ensure maximal efficacy. The different clinical trials completed have adequately demonstrated its efficacy and safety [13-17].

The fact that the treatment with allergy vaccine must be maintained for at least three years in order to get the expected efficacy makes necessary to consider treatment compliance as a relevant variable for the success of the treatment.

Treatment compliance is defined as the process whereby the patient follows treatment with a given drug as prescribed $[18,19]$. In the case of compliance referred to chronic treatment, we also must include the concept of adherence, which is defined as the sum of treatment compliance and its persistence during the indicated period [18]. Different causes can be addressed to the lack of adherence, that have not been fully explored in the case of the treatment with AIT.

In the specific case of treatment adherence in AIT with allergens, Pajno et al. [19] conducted a three-year follow-up study of 2774 patients, of which 1886 received subcutaneous AIT, and found the adherence rate to be $89.1 \%$ versus $78.5 \%$ in the group administered AIT via the sublingual route. It is thought that AIT adherence in usual clinical practice is lower due to different causes including the efficacy that makes the patient think they do not need to continue the treatment.

The present study was carried out to determine patient compliance and adherence to AIT treatment with Allergovit for pollen allergic rhinitis and/or bronchial asthma under real world conditions, up to date unknown in our setting, and explore the main causes for nonadherence.

\section{Materials/methods}

\section{Study design and ethical considerations}

A cross-sectional observational study was designed. The Clinical Research Ethics Committee Inspección General de Sanidad de la Defensa Hospital Gómez Ulla, Madrid, Spain approved the study on 22 December 2015 (reference no. 38/15).

The study was conducted according to the Good Epidemiology Practice guidelines of the International Society for Pharmacoepidemiology, national regulations on observational studies, and basic ethical principles of the Declaration of Helsinki. An electronic case report form was developed, with access limited to the investigators participating in the study.

The retrospective information of the first patient was collected on 16 March 2016 and of the last patient on 21 February 2017. A total of 9 Departments of Allergology belonging to hospitals ascribed to the Spanish National Health System participated in the study.

The study population consisted of patients diagnosed with rhinoconjunctivitis and/or bronchial asthma secondary to pollen allergy and treated with AIT with Allergovit in at least the last three years.

The patients were selected on a retrospective consecutive basis from among all the individuals treated in the past with Allergovit in each center, and who met the following inclusion criteria: 1) Patients of both sexes between 5-65 years of age at the time when AIT was started; 2) Patients diagnosed with rhinoconjunctivitis and/or bronchial asthma of allergic origin; 3) Hypersensitivity to pollen as determined by diagnostic prick testing and/or allergen-specific IgE positivity; 4) Patients already treated with Allergovit' at the time of selection of their case history; and 5) Patients were informed about the study and signed the corresponding informed consent form. For minor patients, parents or legal representative were informed about the study and they also signed the informed consent form.

Patients with a history of treatment with some other allergenic vaccine either simultaneously or in the three years before the administration of Allergovit were excluded, as were those with any contraindication to AIT at the time of administration, as described in the Summary of Product Characteristics [20].

The main study objective was to evaluate adherence to AIT, based on the proportion of patients that abandoned AIT during the three years of the scheduled treatment period. Compliance with AIT was calculated as the proportion of doses administered / doses prescribed during the three years scheduled treatment period, and in each year of treatment. Inadequate compliance was considered when the administered doses totaled less than $80 \%[18,19]$ of the doses that should have been administered in the considered time period.

The secondary objectives were to analyze the main reasons for abandoning AIT, analyze the appearance of adverse events, observe the clinical efficacy of AIT, and assess physician satisfaction with the AIT outcomes at the end of the treatment.

Information was collected referred to patient age and sex, socioeconomic level (low: annual income below 25,000 Euros; medium: annual income between 25,000 and 45,000 Euros; high: annual income above 45,000 Euros), and history of other medical conditions. We recorded the date of the first allergic episode and of the Prick Test [21]. There were also recorded the names of the allergens to which 
the patients were sensitized. As the following data were systematically collected by the participant investigators in the past, the presence of allergic symptoms before the start of AIT was documented, and in each year of follow-up. Also were recorded, ocular or nasal symptoms, bronchial manifestations / asthma, and their weekly frequency divided into four categories coded from $0-3$ as follows: $0=$ absent; $1=$ infrequent ( $\leq 2$ days/week); 2 = frequent ( $>2$ to 5 days/week); and $3=$ very frequent ( $>5$ days/week). The intermittent or persistent nature of the nasal, conjunctival and bronchial symptoms was documented. In turn, the proportion and type of allergen contained in the vaccine and its administration regimen (pre-seasonal, pre-co-seasonal or perennial) were recorded.

The date of the first administered AIT dose was registered, together with the number of doses prescribed each year and the number of doses finally administered. If the administered doses were fewer than the prescribed doses, the reasons for non-compliance or treatment interruption were documented. The possible reasons included improvement of the symptoms, worsening of the symptoms, patient decision with no concrete reason, patient forgetfulness, patient fear of the vaccine, problems in visiting the clinic, financial reasons, the appearance of adverse reactions, or vaccine intolerance. In the event of treatment interruption, the date of interruption was recorded.

At the end of the AIT period, physician Clinical Global Impression referred to patient response was scored based on a visual analog scale (VAS) from $0-10$ points, where $0=$ not at all satisfied, and $10=$ very satisfied.

Likewise, we documented the patient adverse reactions to immunotherapy observed during the treatment period, specifying the start and ending date of the reaction, whether the reaction was immediate (i.e., manifesting in the first 30 minutes after vaccination) or delayed (i.e., $\geq 30$ minutes after vaccination), the treatment measures adopted (none, dose reduction, treatment suspension, drug therapy), and the type of adverse reaction (local or systemic). Systemic reactions were graded from 1-4 [22], and their outcome (resolution, sequelae, death, unknown) was recorded.

\section{Calculation of sample size}

Calculation of the sample size was based on the results of a previous study published by Pajno et al. [19], involving the follow-up of 2692 patients for three years. A total of 1886 of the patients received subcutaneous immunotherapy, and the observed adherence rate was $89.1 \%$. With a sample of 176 patients who received immunotherapy with AIT, a statistical power of $85 \%$ was estimated for determining the percentage of patients showing adherence to immunotherapy for the total period of treatment, with a precision of 0.05 points. Power computation was completed with a normal approximation (arcsine transformation), with an alpha significance level of 0.05 (SPSS, Sample Power).

\section{Statistical analysis}

Data quality control was performed through validation of $100 \%$ of the data and $100 \%$ of the patients.

A descriptive analysis was made, based on the distribution of frequencies of the qualitative variables and calculation of the usual values for continuous variables: mean, standard deviation, minimum and maximum, $95 \%$ confidence interval.

Comparisons between variables were made using the Fisher test or $\mathrm{Chi}^{2}$ test for qualitative variables, while the Student $\mathrm{t}$-test was used for the comparison of independent groups in the case of quantitative variables. Repeated measures analysis of variance (ANOVA) with Bonferroni or Games-Howell correction for multiple comparisons was applied for contrasting the mean frequency of symptoms in the three years of follow-up. Statistical significance was considered for $\mathrm{p}<0.05$. The SPSS version 24.0 statistical package was used throughout.

\section{Results}

A total of 176 patients from 5 Spanish provinces were included in the study, with an average of 19 patients per center (95\%CI 1327). There were 79 males (44.9\%) and 97 females (55.1\%). The mean patient age was 33.8 years (95\%CI 31.9-35.7), with a median of 35 years. The minimum age was 10 years and the maximum 66 years, with no differences between sexes $(\mathrm{p}=0.097)$.

The patient socioeconomic level was medium in 126 (71.6\%), high in $28(15.4 \%)$ and low in $22(12.5 \%)$ of the cases.

The mean duration of allergic disease was 7 years (95\%CI 6-8), with a median of 5 years and no differences between sexes $(\mathrm{p}=0.678)$.

Fifty-one patients (29\%) were sensitized to a single allergen, 70 (39.8\%) were sensitized to two allergens, $11(6.3 \%)$ were sensitized to three allergens, and the remaining $44(24.9 \%)$ were sensitized to more than three allergens. We recorded two patients sensitized to up to 10 different allergens (1.1\%).

\section{Presence and weekly frequency of symptoms}

Seventy-one patients $(40.4 \%)$ presented only rhinoconjunctivitis, while the remaining 105 (59.7\%) presented rhinoconjunctivitis and bronchial / asthma symptoms before AIT.

The nasal symptoms were intermittent in 56 patients (36.8\%) and persistent in 96 (63.2\%). The corresponding information was not available in 24 patients. The bronchial / asthma symptoms in turn were intermittent in 69 patients (69\%) and persistent in 31 (31\%). The corresponding information was not available in 76 patients.

Figure 1 shows the proportion of patients with ocular, nasal and bronchial / asthma symptoms before AIT and over each of the three years of follow-up. The proportion of individuals with symptoms decreased significantly from before treatment (baseline) to the end of the three years of therapy $(\mathrm{p}<0.001)$, with no differences according to sex.

Figure 2 shows the evolution of the weekly frequency of ocular, nasal and bronchial / asthma symptoms, before AIT and over each of the three years of follow-up. The weekly frequency of symptoms was seen to decrease significantly from before AIT (baseline) to the end of

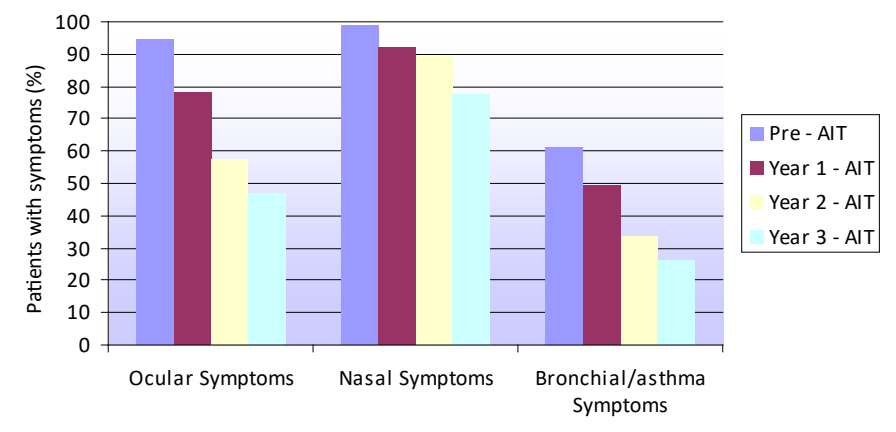

Figure 1. Evolution of the presence of ocular, nasal and bronchial/asthma symptoms before immunotherapy and over each year of AIT treatment follow-up 


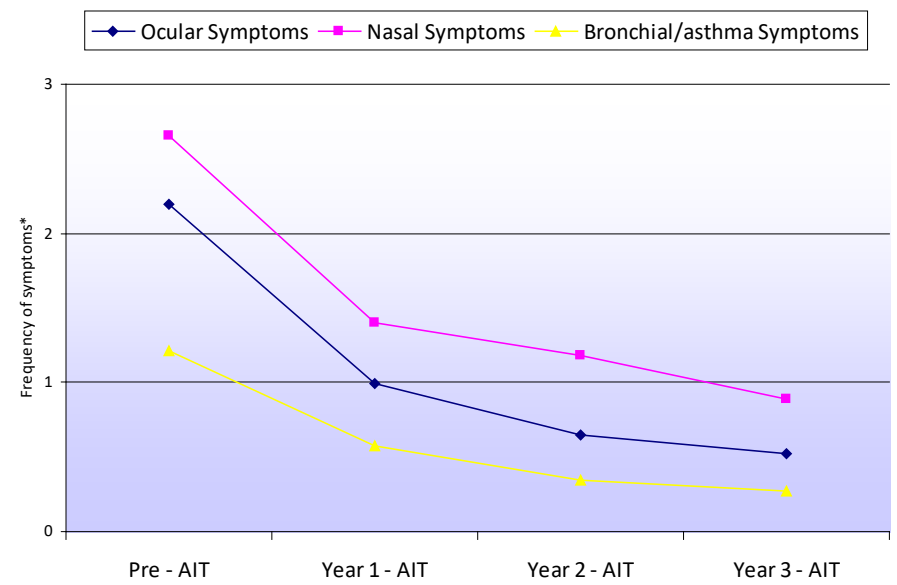

Figure 2. Evolution of the weekly frequency of ocular, nasal and bronchial/asthma symptoms before immunotherapy and over each year of AIT treatment follow-up ("Frequency of symptoms: $0=$ absent; $1=$ infrequent $(\leq 2$ days/week); $2=$ frequent $(>2$ to 5 days/week); $3=$ very frequent $(>5$ days/week)

the first, second and third years of therapy, as well as from the first to the second year, referred to the ocular $(\mathrm{p}<0.0001)$, nasal $(\mathrm{p}<0.0001)$ and bronchial / asthma symptoms $(\mathrm{p}<0.0001)$. The change in weekly frequency between the second and third year failed to reach statistical significance for any of the symptoms. No differences were recorded between males and females.

\section{Composition of Allergovit}

In Table 1 is shown the allergens contained in AIT with Allergovit among the studied patients.

The AIT regimen was pre-seasonal in 32 patients (19.9\%), preco-seasonal in four (2.5\%) and perennial in 125 (77.6\%). The type of treatment regimen was not specified in 15 patients $(8.5 \%)$.

\section{Compliance and adherence to immunotherapy}

An average of 14.1 doses (SD 6.4) of AIT per patient were prescribed during the first year, versus 11.1 doses (SD 2) in the second year and 11.1 doses (SD 2.2) in the third year. A total of 12.1 doses were prescribed on average per year (95\%CI 11.6-12.6).

An average of 13.6 doses (SD 5.4) of AIT with per patient were administered during the first year, versus 10.9 doses (SD 2.4) in the second year and 10.7 doses (SD 2.9) in the third year. A total of 11.8 doses were administered on average per year (95\%CI 11.3-12.2).

Table 2 shows percentage treatment compliance in each year of follow-up according to administration regimen (pre-seasonal, pre-coseasonal, perennial) and total. There were no significant differences between years or between AIT administration regimens. Mean compliance for the three years of immunotherapy was $98 \%$ (95\% CI $96.7-99.3)$. Adequate compliance with the prescribed doses ( $\geq 80 \%)$ was recorded in 163 patients (95.9\%) in the first year, 151 patients (95\%) in the second year and 151 patients $(96.2 \%)$ in the third year.

At the end of each year of AIT, in those cases where the administered doses were fewer than the prescribed doses, the investigators documented the main reason why treatment had not been completed. Table 3 shows the main reasons for non-compliance according to each year of follow-up.

In relation to adherence to therapy, a total of 18 patients (10.6\%) stopped AIT at some point during follow-up (4 patients in the first year,
9 in the second year, and 5 in the third year). Global global adherence was then of $89.8 \%$. The causes of treatment interruption during the first year were the appearance of adverse reactions or vaccine intolerance in three patients and worsening of the symptoms in one patient. In the second year three patients temporarily suspended treatment because of adverse reactions, two patients because of worsening of the symptoms, two more due to financial reasons, one patient because of fear of the vaccine, and one patient due to problems in travelling to the clinic. Lastly, in the third year, four patients interrupted treatment due to nonspecified causes by patient's decision. The patients were able to give one or more reasons for interrupting therapy.

\section{Clinical global impression scale}

At the end of AIT, the mean score of the VAS referred to satisfaction with the clinical response of the patient was 8.23 points out of a maximum of 10 points (95\%CI 8-8.4), with a median of 8 points.

\section{Adverse reactions to immunotherapy}

During the total three-year period we recorded 40 adverse reactions in a total of 34 patients (19.3\%). One case of conjunctivitis,

Table 1. Composition of AIT with Allergovit ${ }^{\circledR}$ in the studied patients

\begin{tabular}{|c|c|c|}
\hline Allergens & N & $\mathbf{\%}$ \\
\hline Artemisia & 3 & 1.9 \\
\hline Grass & 112 & 63.6 \\
\hline Olea europaea & 22 & 13.8 \\
\hline Parietaria & 1 & 0.6 \\
\hline Artemisia-Olea europaea & 3 & 1.9 \\
\hline Grass-Olea europaea & 33 & 20.8 \\
\hline Grass-Parietaria & 1 & 0.6 \\
\hline Phleum-Olea europaea & 1 & 0.6 \\
\hline Total & 176 & 100 \\
\hline
\end{tabular}

Table 2. Percentage treatment compliance (administered doses/prescribed doses $\times 100$ ) in each year of follow-up according to administration regimen

\begin{tabular}{|l|c|c|c|c|c|c|c|c|}
\hline & \multicolumn{4}{|c|}{$\begin{array}{c}\text { AIT administration } \\
\text { regimen* }\end{array}$} & \multicolumn{2}{c|}{$\begin{array}{c}\text { Any administration } \\
\text { regimen }\end{array}$} \\
\cline { 2 - 9 } & $\begin{array}{c}\text { Pre-seasonal } \\
\text { n=32 }\end{array}$ & $\begin{array}{c}\text { Pre-co-seasonal } \\
\text { n=4 }\end{array}$ & $\begin{array}{c}\text { Perennial } \\
\text { n=140 }\end{array}$ & \multicolumn{2}{c|}{$\begin{array}{c}\text { Total } \\
\text { n=176 }\end{array}$} \\
\cline { 2 - 10 } & $\mathbf{M}$ & SD & $\mathbf{M}$ & SD & M & SD & M & SD \\
\hline First year & 99.4 & 3.5 & 100 & 0 & 97.4 & 13.2 & 98.5 & 12.4 \\
\hline Second year & 96.7 & 18.3 & 100 & 0 & 96.2 & 15.5 & 95.9 & 17.2 \\
\hline Third year & 100.0 & 0 & 100 & 0 & 96.2 & 16.6 & 96.6 & 16.2 \\
\hline
\end{tabular}

N: Number of patients; M: Mean; SD: Standard deviation

Table 3. Description of the main reasons for immunotherapy non-compliance according to year of treatment

\begin{tabular}{|c|c|c|c|c|c|c|}
\hline \multirow{2}{*}{ Reason for non-compliance } & \multicolumn{2}{|c|}{ Year 1 } & \multicolumn{2}{c|}{ Year 2 } & \multicolumn{2}{|c|}{ Year 3 } \\
\cline { 2 - 7 } & $\mathbf{n}$ & $\mathbf{9}$ & $\mathbf{n}$ & $\mathbf{\%}$ & $\mathbf{n}$ & $\mathbf{\%}$ \\
\hline Improvement of symptoms & 4 & 25 & 5 & 26.3 & 8 & 57.1 \\
\hline Worsening of symptoms; lack of efficacy & 1 & 6.25 & 2 & 10.5 & 0 & 0 \\
\hline Patient decision with no concrete reason & 1 & 6.25 & 3 & 15.8 & 6 & 42.9 \\
\hline Patient forgetfulness & 1 & 6.25 & 1 & 5.3 & 0 & 0 \\
\hline Patient fear of the vaccine & 0 & 0 & 1 & 5.3 & 0 & 0 \\
\hline Problems travelling to the clinic & 0 & 0 & 1 & 5.3 & 0 & 0 \\
\hline Financial reasons & 0 & 0 & 2 & 0.5 & 0 & 0 \\
\hline $\begin{array}{c}\text { Appearance of adverse reactions or } \\
\text { intolerance to the vaccine }\end{array}$ & 6 & 37.5 & 4 & 21 & 0 & 0 \\
\hline Treatment suspension & 3 & 18.75 & 0 & 0 & 0 & 0 \\
\hline Total & 16 & 100 & 19 & 100 & 14 & 100 \\
\hline
\end{tabular}

$\mathbf{N}$ : Number of patients describing each reason for immunotherapy non-compliance 
33 local reactions, 5 cases of urticaria and one case of asthma were documented. Three were immediate reactions $(7.5 \%)$, while 37 were delayed (92.5\%). Most of the reactions $(n=33,82.5 \%)$ were of a local nature, while 7 were systemic (17.5\%). Three of the systemic reactions were of grade 2 and four of grade 3 [22]. There were no serious adverse reactions. All of these events were resolved and presented a mean duration of 1.7 days (95\% CI 1-2.4).

\section{Discussion}

The World Health Organization, in its position statement on immunotherapy with allergens, defined AIT as the only treatment capable of modifying the natural course of allergic disease and of preventing allergic rhinitis from evolving towards bronchial asthma [5]. The results of our study show adherence to AIT with Allergovit to be high, with very good compliance, with efficacy manifesting from the first year of administration.

An ever-increasing number of publications demonstrate that AIT is already effective in the first pollen season after the start of treatment, or within the first few months in the case of perennial allergens. Matricardi et al. [23] recently published a review comparing different meta-analyses including those with at least 5 randomized, double-blind and placebo-controlled trials, designed to assess the short-term efficacy of both symptomatic drug treatment and AIT. The authors concluded that in the first pollen season after the start of AIT with allergens it was at least as effective as the symptomatic drug treatment available for the patients with allergic rhinitis, as evidenced by the nasal symptoms scores.

We observed a statistically significant $(\mathrm{p}<0.0001)$ decrease in both the presence of ocular, nasal and bronchial / asthma symptoms, and in their weekly frequency. This decrease was already noticeable from the end of the first year of AIT, when maximum symptoms reduction was observed (Figures 1 and 2). However, the decrease remained significant until the end of the second year of AIT, and although symptoms improvement was no longer statistically significant between the second and third year of therapy, the patients continued to derive benefit from the treatment.

Figure 1 shows that at the end of the third year of AIT an important percentage of patients continued to present ocular (46.9\%), nasal $(77.9 \%)$ or bronchial symptoms $(25.9 \%)$, though with a weekly frequency of under two days a week.

In principle, the risk of abandoning treatment once initial efficacy is noted (what we have called non-adherence to therapy), would appear to be greater at the end of the first year of treatment, which is when the greatest decrease in symptoms was observed with respect to the baseline situation before AIT. In our study the adherence rate was $89.4 \%$ in the three years of treatment - which is practically identical to the figure reported by Pajno et al. [19] with subcutaneous AIT (89.1\%) in a large series of patients $(n=1886)$. Four patients abandoned therapy in the first year, 9 in the second year, and 5 in the third year. In this regard, the dropouts recorded in the second year may have been related to the efficacy of AIT, though the analysis of the causes of non-adherence showed no apparent correlation to treatment efficacy.

Studies addressing the adherence to AIT in literature are lacking. We only found one review declaring rates of compliance to subcutaneous AIT of about $70 \%$ where the most common reason for discontinuation was inconvenience, lack of efficacy, costs and loss of working hours [24]. In our study compliance with AIT was very good in all three years of treatment, higher than results of this review. Perhaps data related to efficacy and safety of different AIT products could explain these differences.

The frequency of adverse reactions to AIT in our study cannot be interpreted in true safety terms, particularly in term of the local reactions, and whether these were mild or moderate, since not all such reactions may have been adequately documented. For this reason, the observed proportions may be lower than the real figures. In contrast, the data referred to systemic reactions are presumably more solid, since it is more probable that such reactions were adequately documented in the case histories.

The present study has the limitations that are inherent to retrospective observational studies. Due to the characteristics of these retrospective studies, the collected information may not be so precise. Though when assessing compliance and adherence to immunotherapy, they do afford acceptable data quality, since the patients must visit the Immunotherapy Unit to receive each treatment dose, thereby ensuring registry of the date, dose and relevant adverse reactions. Reasons for noncompliance or non-adherence could not be collected through specific adherence questionnaire due to the retrospective design. The information referred to the weekly frequency of symptoms at the end of each AIT period might not be precise if it is collected retrospectively. But due to this reason, we selected those investigators who systematically collected this efficacy data in their clinical records, and this is a positive aspect of the study. Generalization of the study data to patients with the same characteristics may be considered acceptable.

\section{Conclusions}

In sum, the results of our study indicate that compliance and adherence to subcutaneous immunotherapy (Allergovit) are high, and this data can be used as a reference in our clinical setting.

\section{Declarations}

\section{Ethics approval and consent to participate}

The Clinical Research Ethics Committee Inspección General de Sanidad de la Defensa Hospital Gómez Ulla, Madrid, Spain approved the study on 22 December 2015 (reference no. 38/15).

Thestudy was conducted in line with the GoodEpidemiology Practice guidelines of the International Society for Pharmacoepidemiology (ISPE), national regulations on observational studies, and basic ethical principles of the Declaration of Helsinki.

\section{Availability of data and materials}

The datasets used and analysed during the current study are available from the corresponding author on reasonable request.

\section{Competing interests}

Nataly Cancelliere belongs to the Medical Department of Merck, S.L.U., Spain. Begoña Soler was contracted by Merck, S.L.U., to conduct the design, monitoring, statistical analysis, and medical writing of the publications of the study. All remaining authors received a fee as principal investigators in the study.

\section{Funding}

This work was supported by Merck, S.L.U., Spain. 


\section{Author's contributions}

AMNP, JCH, NC and BSL prepared the design of the study, interpreted the results and prepared the manuscript. Remaining authors read and approved the final manuscript.

\section{Acknowledgements}

We acknowledge the support of the Ethics Committes of the Hospitals: Hospital Universitario Ramón y Cajal, Madrid; Hospital Clínico San Carlos, Madrid; Hospital Universitario Nuestra Señora del Perpetuo Socorro, Albacete; Hospital Rafael Méndez, Lorca (Murcia); Hospital Central de la Defensa Gómez Ulla, Madrid; Hospital Virgen de la Vega, Salamanca; Hospital Universitario La Fe, Valencia; Hospital Universitario Puerta de Hierro, Majadahonda (Madrid); Hospital Virgen del Rocío, Sevilla; Hospital El Tomillar, Sevilla; Fundación Jiménez Díaz, Madrid.

\section{References}

1. Calderon MA, Demoly P, Gerth van Wijk R, Bousquet J, Sheikh A, et al. (2012) EAACI: A European Declaration on Immunotherapy. Designing the future of allergen specific immunotherapy. Clin Transl Allergy 2: 20. [Crossref]

2. Global initiative for asthma (2017) Global strategy for asthma management and prevention. http://www.ginasthma.com.

3. Plaza V, Alonso S, Alvarez C, Gomez-Outes A, Gómez F, et al. (2016) Spanish guideline on the management of asthma. J Investig Allergol Clin Immunol 26: S1S92. [Crossref]

4. Brożek JL, Bousquet J, Agache I, Agarwal A, Bachert C, et al. (2017) Allergic Rhinitis and its Impact on Asthma (ARIA) guidelines-2016 revision. J Allergy Clin Immunol 140: 950-958. [Crossref]

5. Jutel M, Agache I, Bonini S, Burks AW, Calderon M, et al. (2015) International consensus on allergy immunotherapy. J Allergy Clin Immunol 136: 556-568. [Crossref]

6. Abramson M, Puy R, Weiner J (1995) Is allergen immunotherapy effective in asthma? A meta-analysis of randomized controlled trials. Am J Respir Crit Care Med 151: 969974. [Crossref]

7. Abramson M, Puy R, Weiner J (1999) Immunotherapy in asthma: an updated systematic review. Allergy 54: 1022-1041. [Crossref]

8. Abramson M, Puy R, Weiner J (2003) Allergen immunotherapy for asthma. Cochrane Database Syst Rev 2: CD001186. [Crossref]

9. Ross S, Nelson H, Finegold I (2000) Effectiveness of specific immunotherapy in the treatment of asthma: A meta-analysis of prospective, randomized, double-blind placebo-controlled studies. Clin Ther 22: 329-341. [Crossref]
10. Calderon MA, Alves B, Jacobson M, Hurwitz B, Sheikh A, et al. (2007) Allergen injection immunotherapy for seasonal allergic rhinitis. Cochrane Database Syst Rev 24: CD001936. [Crossref]

11. Moreno C, Cuesta-Herranz J, Fernandez-Tavora L, Alvarez Cuesta E on behalf of the SEAIC (2004) Immunotherapy safety: A prospective multicentric monitoring study of biologically standardized therapeutic vaccines for allergic diseases. Clin Exp Allergy 34: 527-31. [Crossref]

12. Eng PA, Borer-Reinhold M, Heijnen IA, Gnehm HP (2006) Twelve-year follow-up after discontinuation of preseasonal grass pollen immunotherapy in childhood. Allergy 61: 198-201. [Crossref]

13. Corrigan CJ, Kettner J, Doemer C, Cromwell O, Narkus A (2005) Efficacy and safety of preseasonal-specific immunotherapy with an aluminium-adsorbed six-grass pollen allergoid. Allergy 60: 801-807. [Crossref]

14. Williams A, Henzgen M, Rajakulasingam K (2007) Additional benefit of a third year of specific grass pollen allergoid immunotherapy in patients with seasonal allergic rhinitis. Eur Ann Allerg Clin Immunol 39: 123-126. [Crossref]

15. Gokmen NM, Ersoy R, Gulbahar O, Ardeniz O, Sin A, et al. (2012) Desensitization effect of preseasonal seven-injection allergoid immunotherapy with olive pollen on basophil activation: The efficacy of olive pollen-specific preseasonal allergoid immunotherapy on basophils. Int Arch Allergy Immunol 159: 75-82. [Crossref]

16. Rajakulasingam K (2012) Early improvement of patients' condition during allergenspecific subcutaneous immunotherapy with a high-dose hypoallergenic 6-grass pollen preparation. Eur Ann Allergy Clin Immunol 44: 128-134. [Crossref]

17. Kahler H, Stüwe H, Cromwell O, Fiebig H (1999) Reactivity of T cells with grass pollen allergen extract and allergoid. Int Arch Allergy Immunol 120: 146-157. [Crossref]

18. WHO (2003) Adherence to long-term therapies. ISBN: 9241545992.

19. Pajno GB, Vita D, Caminiti L, Arrigo T, Lombardo F, et al. (2005) Children's compliance with allergen immunotherapy according to administration routes. $J$ Allergy Clin Immunol 116: 1380-1381. [Crossref]

20. Allergovit, product information. http://www.merck.es/es/compania/divisiones_de merck/biopharma/alergia2/allergovit/allergovit.html.

21. Bousquet J, Heinzerling L, Bachert C, Papadopoulos NG, Bousquet PJ, et al. (2012) Practical guide to skin prick tests in allergy to aeroallergens. Allergy 67: 18-24. [Crossref]

22. Cox L, Larenas-Linnemann D, Lockey RF, Passalacqua G (2010) Speaking the same language: The World Allergy Organization subcutaneous immunotherapy systemic reactions grading system. J Allergy Clin Immunol 125: 569-574. [Crossref]

23. Matricardi PM, Kuna P, Panetta V, Whan U, Narkus A (2011) Subcutaneous immunotherapy and pharmacotherapy in seasonal allergic rhinitis: A comparison based on meta-analyses. J Allergy Clin Immunol 128: 791-799. [Crossref]

24. Senna G, Ridolo E, Calderon M, Lombardi C, Canonica GW, et al. (2009) Evidence of adherence to allergen-specific immunotherapy. Curr Opin Allergy Clin Immunol 9: 544-548. [Crossref]

Copyright: (C2019 Solá Martínez FJ. This is an open-access article distributed under the terms of the Creative Commons Attribution License, which permits unrestricted use, distribution, and reproduction in any medium, provided the original author and source are credited. 